\title{
Final-state effects in high-resolution angle-resolved photoemission from $\mathrm{Ni}(110)$
}

\author{
X. Y. Cui, ${ }^{1, *}$ E. E. Krasovskii, ${ }^{2,3,4}$ V. N. Strocov, ${ }^{1}$ A. Hofmann, ${ }^{5}$ J. Schäfer, ${ }^{5}$ R. Claessen, ${ }^{5}$ and L. Patthey ${ }^{1, \dagger}$ \\ ${ }^{1}$ Swiss Light Source, Paul Scherrer Institute, CH-5232 Villigen, Switzerland \\ ${ }^{2}$ Departamento de Física de Materiales, Facultad de Ciencias Químicas, Universidad del Pais Vasco, 20080 San Sebastián, Spain \\ ${ }^{3}$ Donostia International Physics Center (DIPC), 20018 San Sebastián, Spain \\ ${ }^{4}$ IKERBASQUE, Basque Foundation for Science, 48011 Bilbao, Spain \\ ${ }^{5}$ Experimentelle Physik 4, Universität Würzburg, D-97074 Würzburg, Germany
}

(Received 10 March 2010; revised manuscript received 29 May 2010; published 17 June 2010)

\begin{abstract}
Angle-resolved photoemission with polarized synchrotron radiation is measured from a ferromagnetic single-crystal $\mathrm{Ni}(110)$ surface. Polarization dependence and dispersion with $\mathbf{k}_{\|}$of spectral structures along the high-symmetry lines $\Gamma$-K-X of the bulk Brillouin zone is discussed. An ab initio calculation within the one-step theory of photoemission reveals the relation of the observed structures to initial and final states. The energymomentum distribution of photoemission intensity depending on the light polarization and experimental geometry is explained in terms of even and odd symmetry of the valence-band states with respect to the crystal mirror plane.
\end{abstract}

DOI: 10.1103/PhysRevB.81.245118

PACS number(s): 68.35.B-, 73.20.At, 75.50.Cc, 79.60.-i

\section{INTRODUCTION}

Angle-resolved photoelectron spectroscopy (ARPES) is a powerful experimental tool, which gives access to the spectrum of electronic excitations in crystalline solids with resolution in both energy and wave vector $\mathbf{k}$. The physics of elemental ferromagnetic metals such as $\mathrm{Fe}$ and Ni still poses unsolved questions with regard to their magnetism, the role of interelectronic Coulomb interaction. ${ }^{1,2}$

A case in point is $\mathrm{Ni}$, which has been extensively studied with ARPES, ${ }^{1-3}$ and the measured band structure has been found in a strong disagreement with Kohn-Sham bands calculated within the local spin-density approximation (LSDA). ${ }^{4}$ The experimental width and exchange splitting of the $3 d$ valence bands are, respectively, $\sim 25 \%$ and $\sim 50 \%$ smaller than in LSDA. ${ }^{4,5}$ The quasiparticle GW approximation improves the bandwidth but does not yield the correct exchange splitting. ${ }^{6}$ Many-body calculations based on a combination of GW with the dynamical mean-field theory (DMFT) (Ref. 7) and on Gutzwiller theory ${ }^{8}$ more closely reproduce the experimental data. A calculation of photoemission spectra within the one-step model with the inclusion of correlation effects within the DMFT was performed in Ref. 9 and a striking improvement over the one-particle approach was reported.

Most recently, extensive ARPES studies of the Fermi surfaces (FSs) and energy band dispersions on $\mathrm{Ni}(110)$, Ref. 3, and $\mathrm{Fe}(110)$, Refs. 10-13, single crystals have been performed, in an attempt to identify coupling to spin waves previously observed in these materials with inelastic neutron scattering. ${ }^{14,15}$ Characteristic k-dispersion kinks have been identified and interpreted as spectroscopic signatures of the interaction between the electronic quasiparticles and spin excitations. Thus significant advances in the study of quasiparticle spectra in the presence of bosonic coupling have been made. Nonetheless, regarding the energy-momentum photoemission intensity distribution, even in a noncoupling oneparticle view of the band structure, the role of final state and matrix element effects has remained a poorly addressed aspect.
Here, we report high-resolution ARPES measurements with synchrotron radiation on $\mathrm{Ni}(110)$ with the aim to shed light on the final-state effects. The measurements are performed for two orthogonal light polarizations, which allows us to determine the symmetry of electron states relative to the (110) mirror plane of the surface Brillouin zone (BZ) from the linear dichroism using the dipole selection rules. With a photon energy around $100 \mathrm{eV}$, the conventional freeelectron approximation for the photoemission final states suggests that the experimental spectra reflect the transitions from the initial states at $k_{\perp}=0$, i.e., along the $\Gamma \mathrm{KX}$ surfaceparallel symmetry plane of the BZ. Most of the experimental spectral structures could indeed be assigned to direct transitions from valence bands in the ГKX plane. Surprisingly, we have discovered additional structures with pronounced polarization dependence, which could not be attributed to any bands from this plane or explained by a surface reconstruction or many-body effects. In the following we will call them "ghost" structures.

The interpretation of experimental ARPES data is especially simple for two-dimensional (2D) systems, where the three-dimensional (3D) wave vector $\mathbf{k}$ is reduced to the $2 \mathrm{D}$ component $\mathbf{k}_{\|}$parallel to the surface. The photoemission spectrum $I\left(\epsilon, \mathbf{k}_{\|}\right)$is then the hole spectral function $A\left(\epsilon, \mathbf{k}_{\|}\right)$ weighted with the photoemission cross section as $I\left(\epsilon, \mathbf{k}_{\|}\right)$ $=S\left(\epsilon, E, \mathbf{k}_{\|}\right) A\left(\epsilon, \mathbf{k}_{\|}\right)$, where $S\left(\epsilon, E, \mathbf{k}_{\|}\right)$is the squared matrix element of optical transitions from the initial state of energy $\epsilon$ to the final state of energy $E=\epsilon+\hbar \omega$. If the cross section is a slowly varying function of the arguments, the ARPES signal directly reflects $A(\epsilon, \mathbf{k})$. This picture is often transferred to $3 \mathrm{D}$ systems, where under the assumption of purely direct transitions (strict conservation of crystal momentum) only one $k_{\perp}$ point (neglecting the limited escape depth) contributes to photoemission for a given $\mathbf{k}_{\|}$and photon energy $\hbar \omega$. In fact, an additional complication arises in 3D systems, because the surface breaks the periodicity of the bulk sample in the surface perpendicular direction, so that the momentum $k_{\perp}$ is not a good quantum number any more. This problem has two aspects: (1) the Bloch vector $k_{\perp}$ of the waves in the crystal is different from the wave vector $K_{\perp}$ of the photo- 


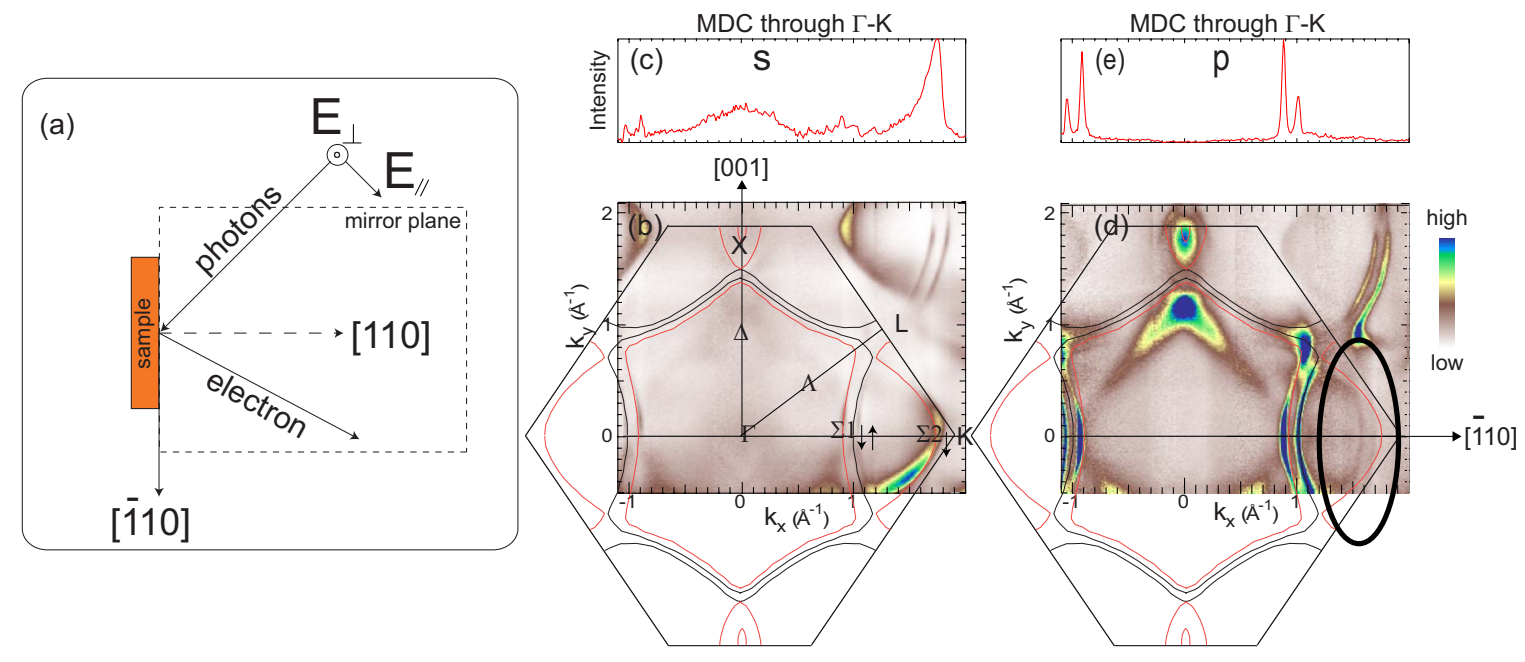

FIG. 1. (Color online) (a) Experimental geometry. The vectors $\mathbf{E}_{\|}$and $\mathbf{E}_{\perp}$ show the electric field of the $p$ - and $s$-polarized incident light, respectively; (b) experimental FSs and (c) MDC at $E_{\mathrm{F}}$ through the $\Gamma \mathrm{KX}$ direction for photon energy $100 \mathrm{eV}$ and $s$ polarization; [(d) and (e)] the same with $p$ polarization; $k_{x}$ is perpendicular and $k_{y}$ is parallel to the experimental plane in (a). Superimposed Gutzwiller calculations of FSs in the $\Gamma$ KX symmetry plane with the thin and thick lines corresponding to the minority and majority bands (Ref. 24).

electron plane wave in vacuum, and, thus, it is not known directly from the experiment. In order to fix $k_{\perp}$ an empirical free-electron approximation for the photoemission final state is often used. However, in many cases this is very inaccurate because even in simple systems final states may significantly deviate from plane waves and their dispersion is far from parabolic. Moreover, the final state may be composed of a few Bloch waves with different $k_{\perp}$ giving comparable contributions to the total photocurrent. ${ }^{16-19}$ In this case $I\left(\epsilon, \mathbf{k}_{\|}\right)$ appears as a combined effect of a few points with different $k_{\perp}$ and $A(\epsilon, \mathbf{k}) ;(2)$ extension of the final state in the surfaceperpendicular direction is limited by the photoelectron meanfree path $\lambda$. This real-space confinement is equivalent to an intrinsic $k_{\perp}$ broadening $\Delta k_{\perp}=\lambda^{-1}$. In this case $I\left(\epsilon, \mathbf{k}_{\|}\right)$reflects an integral of $A(\epsilon, \mathbf{k})$ through the whole $k_{\perp}$ broadening interval rather than $A(\epsilon, \mathbf{k})$ at any particular $k_{\perp}$ point. ${ }^{20,21}$ This fact erodes one of the seemingly fundamental principles of ARPES that the spectral peaks would correspond exactly to direct transitions between the final and the initial state with the same $k_{\perp}$.

Here, we present ARPES data on the Ni(110) ferromagnetic single-crystal surface taken with synchrotron radiation of variable polarization. Interpretation of the experimental data is supported by an ab initio calculation within the onestep theory of photoemission. Our results reveal spectral structures beyond the direct transition model which originate from the final-state broadening characteristic of ARPES from 3D systems.

\section{EXPERIMENTAL PROCEDURE}

The high-resolution ARPES measurements were performed at the Surface and Interface Spectroscopy (SIS) Beamline at the Swiss Light Source (SLS), Paul Scherrer Institute. The end station was equipped with multichannel hemispherical electron-energy analyzers SCIENTA 2002/ $\mathrm{R} 4000$. The total-energy resolution was set to $20 \mathrm{meV}$ in order to achieve good statistics of the spectra. Angular resolution was better than $\Delta \theta=0.2^{\circ}$, which for our photon energies corresponded to $\Delta k \sim 0.01 \AA^{-1}$. The sample was mounted on a low-temperature goniometric manipulator (CARVING) with three angular degrees of freedom and cooled below $10 \mathrm{~K}$. The sample was prepared in situ and measured under ultrahigh vacuum of $1 \times 10^{-11}$ mbar.

The Ni(110) single crystal was cleaned by $\mathrm{Ar}^{+}$-ion sputtering and subsequent annealing at $600{ }^{\circ} \mathrm{C}$ for $30 \mathrm{~min} \cdot{ }^{2,3}$ Cleanliness of the sample surface was checked with angleintegrated photoemission spectra of characteristic core levels. Carbon, nitrogen, oxygen, and sulfur were below the detection limit, confirming that no contaminants existed on the sample surface at low temperature. Low-energy electron diffraction (LEED) demonstrated clear spots in $(1 \times 1)$ pattern, confirming that the surface was well ordered without any reconstructions.

The experimental geometry is shown in Fig. 1(a). The vectors $\mathbf{E}_{\|}$and $\mathbf{E}_{\perp}$ correspond to the electric field of the incident light of $p$ and $s$ polarization, respectively. With different linear polarizations $\mathbf{e}$, the dipole selection rules can be exploited to determine the symmetry of states with respect to the mirror planes of the crystal surface. In the case of emission in the crystal mirror plane, the final state $\Phi$ is even with respect to the symmetry operations of the plane. In order, for the dipole matrix element $\langle\Phi|\mathbf{e} \cdot \mathbf{p}| \Psi\rangle$ to be nonzero, the initial state $\Psi$ must have the symmetry of the dipole operator $\mathbf{e} \cdot \mathbf{p}$. Therefore, if $\mathbf{e}$ lies in the collection mirror plane ( $p$ polarization) then $\Psi$ must be symmetric (even), whereas if $\mathbf{e}$ is perpendicular to the mirror plane ( $s$ polarization) then $\Psi$ must be antisymmetric (odd).

Our experimental data were acquired with a photon energy of $\hbar \omega=100 \mathrm{eV}$. The components of the photoelectron wave vector parallel to the sample surface, $k_{\|}$, was determined by the equation $k_{\|}=\sqrt{2 m E_{\mathrm{k}} / \hbar^{2}} \sin \theta$, where $E_{\mathrm{k}}$ is the photoelectron kinetic energy and $\theta$ is the angle relative to the surface normal. As the first approximation, the perpendicular component $k_{\perp}$ was determined from the free-electron ap- 
proximation for the final states as $k_{\perp}$ $=\sqrt{2 m\left(E_{\mathrm{k}} \cos ^{2} \theta+V_{0}\right) / \hbar^{2}}$, where $V_{0}$ is the inner potential. ${ }^{22}$ On the basis of results obtained by previous ARPES measurements ${ }^{2,23}$ we assumed $V_{0}=10.7 \mathrm{eV}$. The photon energy $\hbar \omega=100 \mathrm{eV}$ was chosen so that for $k_{\|} \sim 1.75 \AA^{-1}$, where the $\Sigma_{2 \downarrow}$ band crosses the Fermi level, $k_{\perp}$ would appear at the $\Gamma \mathrm{KX}$ symmetry line of the third $\mathrm{BZ}\left(k_{\perp}=0\right.$ in the reduced zone). To verify this choice, we have checked the $k_{\|}$ location of the $\Sigma_{2 \downarrow}$ band by measuring the Fermi-surface arc around $k_{\perp}=0$ and $k_{\|}=1.75 \AA^{-1}$ under variation in photon energy. The extremal $k_{\|}$location of the intensity maximum, which corresponds to the emission from the $\Sigma_{2 \downarrow}$ band, occurred indeed at $100 \pm 3 \mathrm{eV}$.

\section{EXPERIMENTAL RESULTS}

The experimental FSs and the momentum distribution curves (MDCs) at $E_{\mathrm{F}}$ corresponding roughly to the $\Gamma \mathrm{KX}$ direction are shown in Figs. 1(b) and 1(c) for $s$ polarization and Figs. 1(d) and 1(e) for $p$ polarization. (Strictly speaking, our path in $k$ space as a function of $k_{\|}$does not exactly coincide with the $\Gamma \mathrm{KX}$ direction because for a fixed photon energy $k_{\perp}$ depends on $k_{\|}$. However, to simplify our discussion, we refer to it as ГKX.) Based on direct transitions between the initial and final bands (under quasiconservation of the 3D $\mathbf{k}$ vector, taking into account the effect of the inner potential $V_{0}$ on $k_{\perp}$ ), the structures in the experimental data can be identified by comparison with the results of the Gutzwiller band-structure calculations ${ }^{24}$ in Figs. 1(b) and $1(\mathrm{~d})$, with the thin and thick curves displaying the minority and majority FS. The $\Sigma_{2 \downarrow}$ bulk-derived band is visible along the $\Gamma \mathrm{K}$ direction in the case of $s$ polarization and it is completely absent in the case of $p$ polarization. On the contrary, the $\Sigma_{1 \uparrow \downarrow}$ bands along $\Gamma \mathrm{K}, \Delta$ bands along $\Gamma \mathrm{X}$, and $\Lambda$ bands along $\Gamma \mathrm{L}$ are visible for $p$ polarization, and lose their intensity for $s$ polarization. Here we only focus on $\Sigma$ bands, but not $\Delta$ and $\Lambda$ bands, because the measurement plane of the latter bands is away from the mirror plane. They do not disappear completely, which identifies strong matrix-element effects different from symmetry selection rules. ${ }^{25}$ The dispersion of these bands well agrees with the calculated FSs. ${ }^{24}$

Besides the above ARPES structures identified with certain bands, our data show two faint ghost bands (with intensity at the percent level compared to regular bands) at $k_{\|}$ $=1.56$ and $1.68 \AA^{-1}$ along $\Gamma \mathrm{K}$ direction, noted with thick ellipse in Fig. 1(e). They are most pronounced for $p$ polarization and have lower intensities in $s$ polarization.

The polarization dependence of the experimental data is illustrated in Fig. 2. The intensity maps and $E_{\mathrm{F}}$ MDCs for the $\Gamma \mathrm{KX}$ direction measured with $s$ and $p$ polarization are shown in Figs. 2(a) and 2(b), respectively. The $\Sigma_{2 \downarrow}$ band gains high intensity for $s$ and almost disappears for $p$ polarization. Intensity of the $\Sigma_{1 \uparrow \downarrow}$ bands, on the contrary, is high for $p$ and small for $s$. The two parallel ghost bands [dashed lines in Figs. 2(b) and 2(c)] are most intense for $p$ polarization, which suggests that their wave functions are similar to the $\Sigma_{1 \uparrow \downarrow}$ bands rather than to $\Sigma_{2 \downarrow}$. Figures 2(c) and 2(d) show the same data measured with circular-left (CL) and circular-right (CR) light. As the circular polarization includes $s$ and $p$ con-

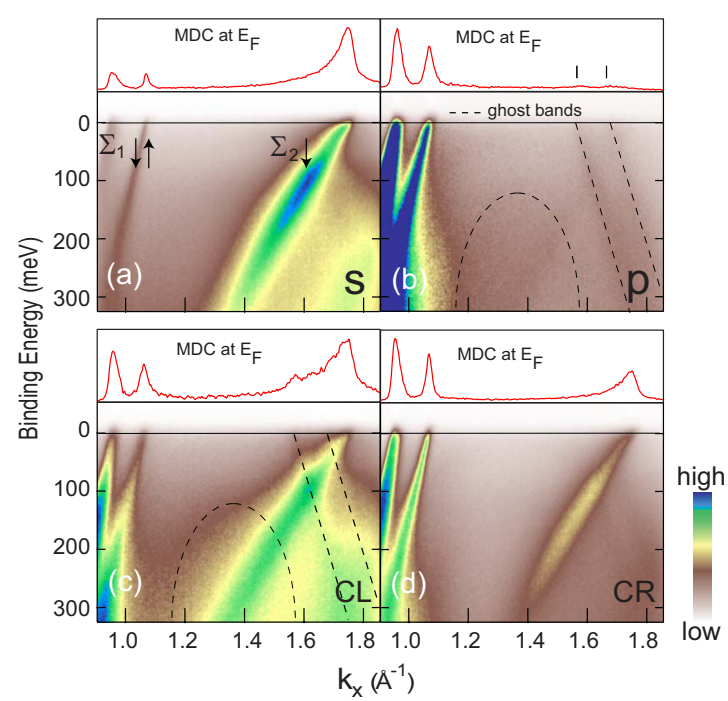

FIG. 2. (Color online) MDCs at $E_{\mathrm{F}}$ and energy intensity plots for $\hbar \omega=100 \mathrm{eV}$ along $\Gamma \mathrm{K}$ at $10 \mathrm{~K}$ measured with (a) $s$, (b) $p$, (c) left, and (d) right circular polarization. Dashed lines in graphs (b) and (c) show the ghost bands.

tributions, the above $\Sigma_{1 \uparrow \downarrow}, \Sigma_{2 \downarrow}$ and ghost bands are visible for both chiralities. However, their intensity shows strong dichroism due to different matrix elements. ${ }^{22}$ The ghost bands are stronger for CL. Upon increase in $k_{\|}$they disperse from $E_{\mathrm{F}}$ downwards, overlap with the $\Sigma_{2 \downarrow}$ at a binding energy of $120 \pm 20 \mathrm{meV}$, and then merge together starting from $300 \mathrm{meV}$. The ghost bands lose their intensity for CR light.

The origin of the ghost bands is obviously beyond the usual direct transitions picture. Furthermore, we can rule out any surface states or resonances as possible origin of these structures because our recent ARPES measurements under gas adsorption have confirmed the absence of such states for the clean $\mathrm{Ni}(110)$ surface. Any superstructure origin can also be ruled out based on LEED patterns observed for the clean surface, Fig. 3(a). Sharp spots without any sign of superstructure indicate that there is no reconstruction either on the surface or in the bulk, at least within the LEED probing depth which is close to the photoelectron escape depth. This is consistent with results of previous scanning tunneling microscope experiments on $\mathrm{Ni}^{26}$ which have also found no reconstructions. Finally, we have investigated evolution of the ghost bands under surface adsorption of oxygen. The LEED pattern after deposition of $\mathrm{O}_{2}$ in a dose of $10 \mathrm{~L}$ is displayed in Fig. 3(b). It shows that the chemisorbed oxygen starts to form ordered overlayers resulting in $(2 \times 1)$-O surface reconstruction. ${ }^{27}$ The $E_{\mathrm{F}} \mathrm{MDCs}$ through the $\Gamma \mathrm{K}$ direction measured for the clean and $(2 \times 1)-\mathrm{O}$ surfaces are displayed in Figs. 3(c) and 3(d), and the corresponding intensity maps in Figs. 3(e) and 3(f). After the oxygen absorption the two ghost bands completely vanish. This fact indicates that the $(2 \times 1)$-O surface reconstruction breaks the old symmetry and eliminates the ghost structures. In Sec. IV we demonstrate that the ghost bands have in fact their origin in finalstate effects at the clean (110) surface.

Another less obvious point of our experimental data is that the $\Sigma_{2 \downarrow}$ band retains notable width even at $E_{\mathrm{F}}$. Com- 

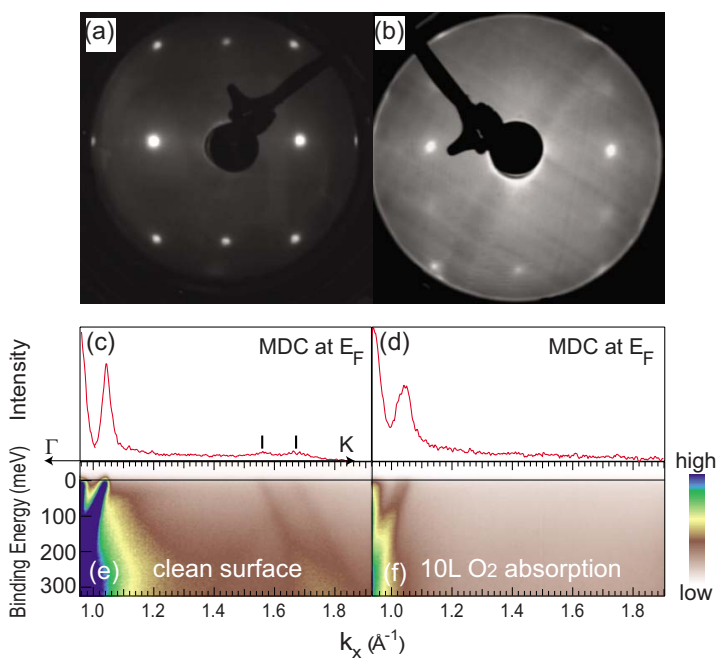

FIG. 3. (Color online) (a) Sharp LEED $(1 \times 1)$ pattern of clean $\mathrm{Ni}(110),(\mathrm{b})$ the $(2 \times 1)$-oxygen reconstructed surface of the chemisorbed $\mathrm{O} / \mathrm{Ni}(110)$. The MDC at $E_{\mathrm{F}}$ and intensity plots measured through $\Gamma$-K direction for clean (c) and (e) and chemisorbed (d) and (f) surface.

monly, the photoemission linewidths $\Gamma_{\text {tot }}$ for 3D systems are analyzed with the expression $\Gamma_{\text {tot }} \approx \Gamma_{h}+\Gamma_{e}\left|V_{h \perp}\right| /\left|V_{e \perp}\right|$, where $\Gamma_{h}$ and $\Gamma_{e}$ are the lifetime broadening of photohole and photoelectron, respectively, and $V_{h \perp}$ and $V_{e \perp}$ are their surfaceperpendicular group velocities. ${ }^{22}$ Our choice of photon energy forces the $\Sigma_{2 \downarrow}$ band to reach $E_{\mathrm{F}}$ when its $k_{\perp}$ is placed at the $\Gamma \mathrm{KX}$ symmetry line, delivering the extremal point of its dispersion and, thus, $V_{h \perp}=0$. Then, in principle, $\Gamma_{\text {tot }}$ should reduce to solely $\Gamma_{h}$ and vanish at $E_{\mathrm{F}}$. This obviously disagrees with our experimental data. An explanation of this effect, besides certain contributions due to scattering between electrons and impurities, goes back to intrinsic broadening of the final states in $k_{\perp} \cdot{ }^{18,20,21}$ Even when $k_{\perp}$ is placed on the symmetry line, contributions from other states within the $k_{\perp}$ broadening interval away from the symmetry line result in nonvanishing $\Gamma_{\text {tot }}{ }^{21}$ The above common expression for $\Gamma_{\text {tot }}$ cannot properly describe this situation, as it implies replacement of the final-state $k_{\perp}$ broadening by artificial energy broadening, which is valid only under assumption of linear band dispersion. ${ }^{21}$

\section{ONE-STEP PHOTOEMISSION ANALYSIS}

We shall now consider the role of final states in photoemission from $\mathrm{Ni}(110)$, in particular, the effect of the surface-perpendicular momentum conservation. Our aim is to explain the experimentally observed structures in terms of the Bloch-wave structure of the outgoing photoelectron wave based on the one-step photoemission theory. ${ }^{28}$

According to DMFT calculations, ${ }^{7,9}$ in Ni the correlation effects shift the bands by as much as $0.5 \mathrm{eV}$ from their LSDA positions. At the same time, the overall shape of the $\epsilon(\mathbf{k})$ lines in DMFT remained practically the same as in LSDA. Because DMFT is beyond our computational abilities, in this analysis we leave aside the question of the actual spectral function of $\mathrm{Ni}$ and concentrate on how its gross fea-

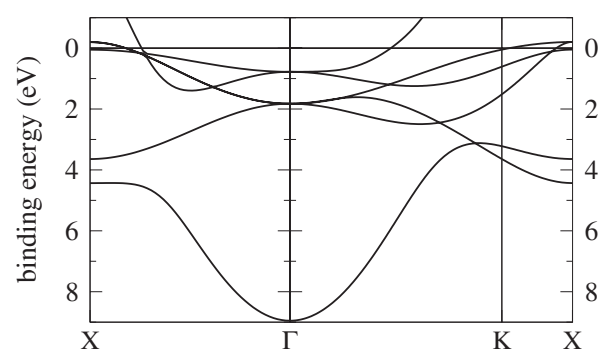

FIG. 4. Reference band structure of Ni calculated within a selfconsistent nonspin-polarized LDA.

tures manifest themselves in the experimental energymomentum intensity distribution. For simplicity, we base our analysis on a single-particle nonspin-polarized local-density approximation (LDA) band structure, Fig. 4, which may serve as a reference model for each of the spin-split bands. The self-consistent potential of the bulk crystal was obtained with the full-potential augmented plane-waves method, ${ }^{29}$ and the calculation of photoemission was performed for a semiinfinite crystal with a steplike surface barrier, as explained in the following.

\section{A. Description of initial and final states}

Because of the broken translational invariance in the surface perpendicular direction the initial states are superpositions of Bloch waves. In the bulk of the crystal the initial state is a standing wave (in the surface perpendicular direction) composed of a Bloch wave incident from the interior of the crystal on the surface and one or more reflected waves. ${ }^{17}$ In the close vicinity of the surface the wave function is strongly modified due to the reflection from the crystalvacuum potential barrier, and it cannot, in general, be represented by a superposition of propagating Bloch waves. However, if the bulk contribution to the excitation matrix element dominates the contribution from the surface region, the spectral intensity can be expressed in a form that provides a rather clear connection to the underlying bulk band structure $\epsilon(\mathbf{k})$. The energy distribution of the emission intensity at a given $\mathbf{k}_{\|}$and photon energy $\hbar \omega$ is an integral over the initial states,

$$
I\left(\mathbf{k}_{\|}, \epsilon\right)=\sum_{m} \int d k_{\perp} S_{m}\left(\mathbf{k}_{\|}, k_{\perp}\right) \delta\left[\epsilon_{m}\left(\mathbf{k}_{\|}, k_{\perp}\right)-\epsilon\right] .
$$

Here $m$ is the band index of the incident wave, which together with its Bloch vector $\mathbf{k}=\left(\mathbf{k}_{\|}, k_{\perp}\right)$ naturally labels the initial state. The cross section $S_{m}\left(\mathbf{k}_{\|}, k_{\perp}\right)$ is the squared modulus of the dipole matrix element between the initial state $\Psi$ of energy $\epsilon$ and final state $\Phi$ of energy $E=\epsilon+\hbar \omega$,

$$
S_{m}\left(\mathbf{k}_{\|}, k_{\perp}\right)=\left|\left\langle\Phi\left(E, \mathbf{k}_{\|}\right)|\mathbf{e} \cdot \mathbf{p}| \Psi_{m}\left(\mathbf{k}_{\|}, k_{\perp}\right)\right\rangle\right|^{2} .
$$

The initial state $\left|\Psi_{m}\right\rangle$ is a linear combination of the Bloch eigenfunctions $\left|k_{\perp n}\right\rangle$ of the bulk Hamiltonian for the same energy $\epsilon$. In other words, $\left|k_{\perp n}\right\rangle$ are the solutions of the inverse band-structure problem $\epsilon_{n}\left(k_{\perp n}\right)=\epsilon$, see Ref. 30 .

In the one-step photoemission theory, ${ }^{28}$ the final state $|\Phi\rangle$ is the time-reversed LEED state defined by the Bloch vector 

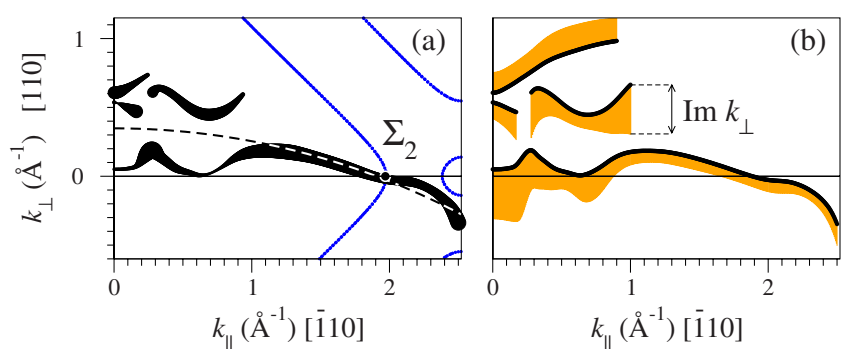

FIG. 5. (Color online) CBS decomposition of LEED states at $E=100 \mathrm{eV}$ for $\mathbf{k}_{\|} \|[\overline{1} 10]$ and $\mathbf{k}_{\perp} \|[110]$ shown by constant energy contours $\operatorname{Re} \kappa_{\perp}\left(k_{\|}\right)$for the Bloch waves that most strongly contribute to the LEED state. (a) Thickness shows the partial current carried by the waves. Dashed curve is the free-electron approximation, see end of Sec. II. Full lines are constant energy contours $\epsilon\left(k_{\|}, k_{\perp}\right)=0$ for odd initial states. (b) Vertical extent of the shaded area shows the momentum broadening $\operatorname{Im} \kappa_{\perp}\left(k_{\|}\right)$.

$\mathbf{k}_{\|}$and energy $E$. (The LEED wave function is a scattering solution for a plane wave incident from vacuum.) The LEED state incorporates the effects of inelastic scattering of the photoelectron, which are quantitatively treated by adding an imaginary part $-i V_{\mathrm{i}}$ (optical potential) to the potential in the crystal half space. ${ }^{31}$ Thus, $|\Phi\rangle$ is an eigenfunction of a nonHermitian Hamiltonian with a real eigenvalue $E$. The term $-i V_{\mathrm{i}}$ governs the spatial decay of the function $|\Phi\rangle$ into bulk, which is responsible for the surface sensitivity of photoemission in the one-step model. In the crystal the LEED function is given by its partial waves (complex band structure, CBS) expansion: $|\Phi\rangle=\Sigma\left|\kappa_{\perp j}\right\rangle$, each partial wave $\left|\kappa_{\perp j}\right\rangle$ being a Bloch solution of the Schrödinger equation with a complex surface-normal projection $\kappa_{\perp j}$ of the wave vector. ${ }^{32}$ Relative contributions of the Bloch waves $\left|\kappa_{\perp}\right\rangle$ can be characterized by the partial currents $T_{\kappa}$ transmitted by the waves into the crystal in the LEED experiment. In the presence of the optical potential all $\kappa_{\perp j}$ have a nonzero imaginary part and the transmitted current is zero. However, the current conservation law can be generalized to the case of absorbing potential ${ }^{33}$ and the partial weights are given by individual absorbed currents, see Fig. 5(a). In the present work, the functions $|\Phi\rangle$ are calculated from the complex band structure of the semi-infinite crystal with the inverse $\mathbf{k} \cdot \mathbf{p}$ method in the extended linear augmented plane-wave formalism. ${ }^{30}$ The absorbing potential was taken to be $V_{\mathrm{i}}=2 \mathrm{eV}$.

Although the LDA potential is a rather poor approximation for the quasiparticle self-energy at high energies the LDA band structure is known to be practically uniformly shifted relative to the true one. In the present calculation we rely on the experimentally determined final state in the freeelectron approximation (see the last paragraph of Sec. II): the ab initio LDA bands were shifted by $3 \mathrm{eV}$ upwards so that at $E=100 \mathrm{eV}$ the main LEED-state constituent intersects the $\Gamma K X$ line close to the position of the $\Sigma_{2}$ band at $\epsilon=0$ [see the curve of variable thickness in Fig. 5(a)]. The value of $3 \mathrm{eV}$ agrees well with our previous experience: at high energies similar values of the self-energy shift have been observed for $\mathrm{TiTe}_{2}$ (Ref. 18) and for aluminum. ${ }^{19}$ Note that for $k_{\|}$ $<1 \AA^{-1}$ the true LEED state strongly deviates from the freeelectron approximation [dashed line in Fig. 5(a)]: two Bloch
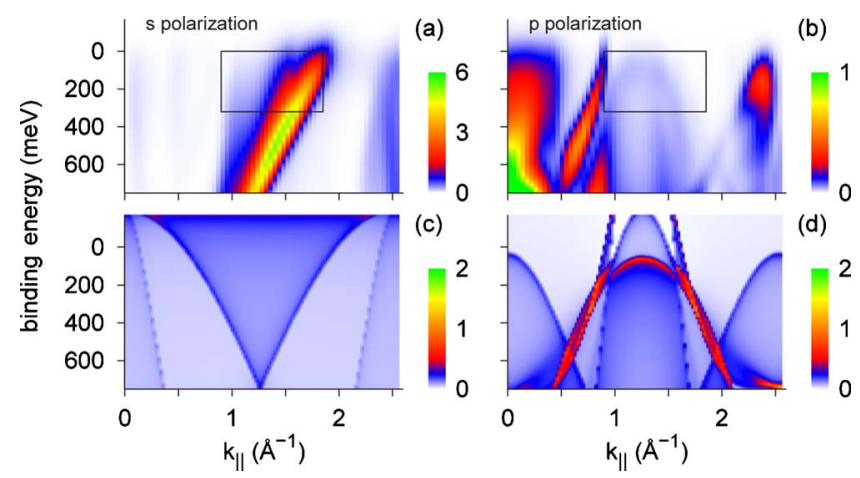

FIG. 6. (Color online) Calculated photoemission intensity $I\left(k_{\|}, E\right)$ distribution for $\mathbf{k}_{\|}$along $[\overline{1} 10]$ and $\hbar \omega=100 \mathrm{eV}$. Intensity distributions (a) for $s$ and (b) for $p$ polarization are compared to the $\mathbf{k}_{\|}$projected DOS (c) for odd and (d) for even states. The boxes in graphs (a) and (b) show the range of the measurements in Fig. 2.

waves with different $\kappa_{\perp}$ equally strongly contribute to the final state. Figure 5(b) shows that the momentum broadening $\operatorname{Im} \kappa_{\perp}$ is different for different waves and that it strongly varies with $k_{\|}$.

\section{B. $\mathbf{k}_{\|}$-projected DOS and ARPES}

The calculated energy-momentum intensity distributions $I\left(\mathbf{k}_{\|}, \epsilon\right)$ from $\mathrm{Ni}(110)$ are shown in Fig. 6(a) ( $s$ polarization) and in Fig. 6(b) ( $p$ polarization). The inverse lifetime of initial states was taken to be $20 \mathrm{meV}$ so the width of the EDC peaks comes from the final-state broadening. In spite of the LDA approximation and the neglect of the exchange splitting, the overall intensity distribution is very similar to the measured one, see Figs. 2(a) and 2(b).

Regarding the effect of final states on the energymomentum intensity distribution, two limiting cases may occur, depending on the strength of inelastic scattering. First, if the inelastic effects are negligible the photoelectron meanfree path is large (for propagating waves $\operatorname{Im} \kappa_{\perp j}$ are small). The energy location of the spectral peaks and their dispersion with $\mathbf{k}_{\|}$is then determined by the energy dependence of the cross section, which has sharp peaks at the energies that satisfy the crystal momentum-conservation requirement,

$$
S_{m}\left(\mathbf{k}_{\|}, \boldsymbol{\epsilon}\right) \sim \sum_{n j} \delta\left[k_{\perp n}\left(\mathbf{k}_{\|}, \boldsymbol{\epsilon}\right)-\kappa_{\perp j}\right],
$$

i.e., main spectral features come from direct transitions, which is typical of simple metals, e.g., aluminum. ${ }^{19}$

In the other limiting case, the transitions are entirely indirect (all partial waves are strongly evanescent; Im $\kappa_{\perp j}$ are large), and the final states do not play a strong selective role $[\delta$ functions in Eq. (3) are smeared out]. Then the photoemission intensity $I\left(\mathbf{k}_{\|}, \boldsymbol{\epsilon}\right)$ is proportional to the $\mathbf{k}_{\|}$-projected density of states (DOS),

$$
\rho\left(\mathbf{k}_{\|}, \epsilon\right)=\sum_{m} \int d k_{\perp} \delta\left[\epsilon_{m}\left(\mathbf{k}_{\|}, k_{\perp}\right)-\epsilon\right] .
$$

The $\mathbf{k}_{\|}$-projected DOS $\rho\left(\mathbf{k}_{\|}, \boldsymbol{\epsilon}\right)$ in the vicinity of the Fermi level is shown in Figs. 6(c) and 6(d) for odd (seen in $s$ 


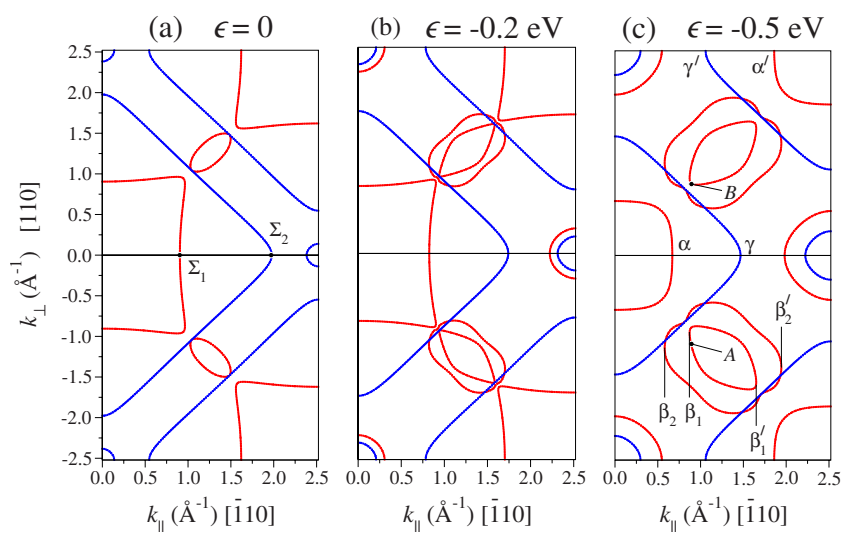

FIG. 7. (Color online) Constant energy lines $\epsilon\left(k_{\|}, k_{\perp}\right)=$ const for $\mathbf{k}_{\|} \|[\overline{1} 10]$ and $\mathbf{k}_{\perp} \|[110]$ (a) for $\epsilon=0$, (b) for -0.2 , and (c) for $-0.5 \mathrm{eV}$ relative to the Fermi energy. Even (odd) states are shown by red (blue) lines.

polarization) and even ( $p$ polarization) states, respectively. Because the DOS function (integrated over the crystal volume) is a bulk property, and the $\mathbf{k}_{\|}$and $\mathbf{k}_{\perp}$ directions are related by a point-group symmetry operation, the $\rho\left(k_{\|}, E\right)$ functions are symmetric relative to $k_{\|}=\sqrt{2} \pi / a=1.26 \AA^{-1}(a$ is the fcc lattice constant). The maxima of the $\mathbf{k}_{\|}$-projected DOS $\rho$ are related to the structure of the constant energy contours $\epsilon\left(k_{\|}, k_{\perp}\right)=$ const (the cut $\epsilon=0$ is the Fermi surface), see Fig. 7. They correspond to the points at which the tangent to the contour is parallel to the $k_{\perp}$ axis [indicated by Greek letters in Fig. 7(c)]. In particular, the points $\alpha, \alpha^{\prime}$ and $\beta_{1,2}, \beta_{1,2}$ give rise to the DOS maxima lines in Fig. 6(d) going up as $k_{\|}$approaches the center $k_{\|}=\sqrt{2} \pi / a$. The most intense lines come from the distorted squares $\alpha$ and $\alpha^{\prime}$, and the narrow inverted parabola comes from the rugby-ballshaped contour $\beta_{1}-\beta_{1}^{\prime}$. The wider parabola due to the $\beta_{2}-\beta_{2}^{\prime}$ contour overlaps with the $\alpha$ - $\alpha^{\prime}$ lines making the two structures difficult to separate below $200 \mathrm{meV}$.

In the calculated $I\left(\mathbf{k}_{\|}, \epsilon\right)$ map for $p$ polarization [Fig. 6(b)] a symmetric structure coming from the $\beta_{1}-\beta_{1}^{\prime}$ parabola is well visible. Thus, we can interpret the experimentally observed weak arclike structure and the spin-split ghost band at $k_{\|}=1.56$ and $1.68 \AA^{-1}$ shown by dashed lines in Fig. 2(b) as coming from the contours $\beta_{1}-\beta_{1}^{\prime}$ and $\beta_{2}-\beta_{2}^{\prime}$ in Fig. 7 centered at $k_{\|}=k_{\perp}=\sqrt{2} \pi / a$. It should be noted that the $\alpha^{\prime}$ line may contribute to the weak structure as well. However, in the experiment, the strong $\Sigma_{1}$ structures at the Fermi energy appear at $k_{\|}=0.96$ and $1.07 \AA^{-1}$, see Fig. 2(b), so their symmetry counterparts due to the $\alpha^{\prime}$ ghost should appear at $k_{\|}$ $=1.45$ and $1.56 \AA^{-1}$, i.e., about $0.1 \AA^{-1}$ to the left from the observed ghosts. Therefore the main contribution to the observed ghost structures appears to come from the contours $\beta_{1}-\beta_{1}^{\prime}$ and $\beta_{2}-\beta_{2}^{\prime}$.

Also in the calculation, the $\alpha$ branch of the DOS function produces a strongly asymmetric intensity distribution: its left branch has very high emission intensity, whereas the $\alpha^{\prime}$ branch is strongly suppressed, in agreement with the experiment. The same happens to the $\gamma$ and $\gamma^{\prime}$ parabolas of odd states, centered at $\Gamma$ in the first and second BZ, respectively. Again, in $s$ polarization we see the intensity peak dispersing toward Fermi energy as the $k_{\|}$grows, and not its symmetry counterpart for the diminishing $k_{\|}$. This perfectly agrees with the experiment [compare Figs. 2(a) and 6(a)].

We see that some structures coming from the peaks of $\mathbf{k}_{\|}$-projected DOS are well visible in the intensity map in spirit of the indirect-transitions picture, whereas the other ones disappear manifesting the effect of momentum conservation. This behavior can be understood from Fig. 7: in scanning along the $\mathbf{k}_{\|}$direction the $k_{\perp}$ components of the initial states vary to form constant-energy contours of very different size. Thereby varies the distance $\Delta k_{\perp}$ from the initial-state Bloch vectors to the final-state ones, which causes more or less strong intensity variations depending on how important the momentum conservation is. The latter is contained in the transition matrix element and the transition intensity behaves qualitatively as a Lorentzian function of $\Delta k_{\perp}$ with the half width equal to $\operatorname{Im} \kappa_{\perp}$ : it is sharply peaked at $\Delta k_{\perp}=0$ and decays rather slowly at $\Delta k_{\perp}$ larger than $\operatorname{Im} \kappa_{\perp}$ [which for the main branch is on the order of $0.1 \AA^{-1}$, as shown in Fig. 5(b)].

Note that for the whole interval of $k_{\|}$the wave vector $\kappa_{\perp}$ of the main final-state branch at $E=100 \mathrm{eV}$ remains close to $k_{\perp}=0$, see Fig. 5. Thus, we arrive at the following qualitative explanation of the ARPES map: because $\beta_{1,2}$ and $\beta_{1,2}^{\prime}$ are close to each other in $k_{\perp}$ (and they both are rather far from $k_{\perp}=0$ compared to $\operatorname{Im} \kappa_{\perp}$, i.e., transitions are indirect) the selective effect of the final states is reduced and we see the whole inverted parabola in the $I\left(k_{\|}, \epsilon\right)$ map in Fig. 6(b). On the contrary, the peaks from $\alpha$ and $\gamma$ come from $k_{\perp}=0$ (direct transitions), whereas $\alpha^{\prime}$ and $\gamma^{\prime}$ are at the edge of the one-dimensional BZ, so the latter are strongly suppressed in ARPES because they are too far from the $k_{\perp}$ of the final state.

\section{C. k-space anatomy of transitions}

Although the above qualitative consideration already sheds light on the origin of the ghost bands, it is instructive to have a closer look at the role of the final states in the formation of the intensity distribution. As an example, Fig. 8 presents a reciprocal-space analysis of the dipole transition amplitude from two initial states, whose incident waves [labeled A and B in Fig. 7(c)] come from the rugby-ball-shaped circles at $\epsilon=-0.5 \mathrm{eV}$. The two states form the inner parabola in Fig. 6(d).

The structure of the final states for several $\mathbf{k}_{\|}$in the energy interval $93-105 \mathrm{eV}$ is presented in Fig. 8(a). The distribution of the absorbed current $T_{\kappa}$ over the partial waves is shown by the thickness of the $E\left(\operatorname{Re} \kappa_{\perp}\right)$ curves. The character of the LEED state is seen to strongly depend not only on $k_{\|}$but also on the energy. However, with $k_{\|}$moving away from $\bar{\Gamma}$ the LEED state turns more "free-electronlike:" the central branch (smallest Re $\kappa_{\perp}$ ) becomes strongly dominating [see also Fig. 5(a), in which the data for $E=100 \mathrm{eV}$ are summarized].

Figures $8(\mathrm{~b})$ and $8(\mathrm{c})$ show the partial transition amplitudes $\mu_{A, B}\left(\kappa_{\perp}\right)=\left\langle\kappa_{\perp}|\mathbf{e} \cdot \mathbf{p}| \Psi_{A, B}\right\rangle$ to each of the final-state waves $\left|\kappa_{\perp}\right\rangle$. These values characterize the contributions from the individual Bloch waves to the photocurrent. The transi- 
(a) current

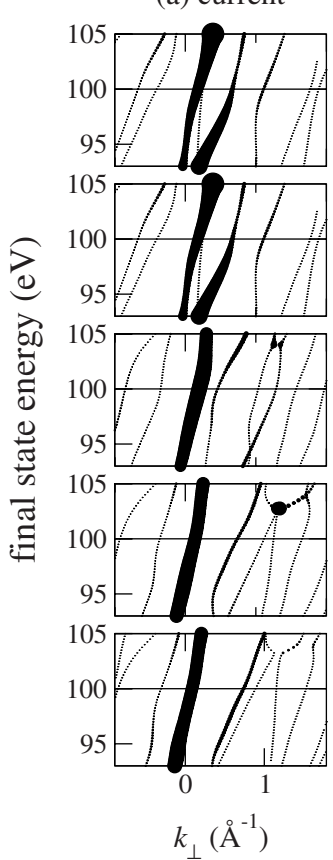

(b) $\mu_{A}$
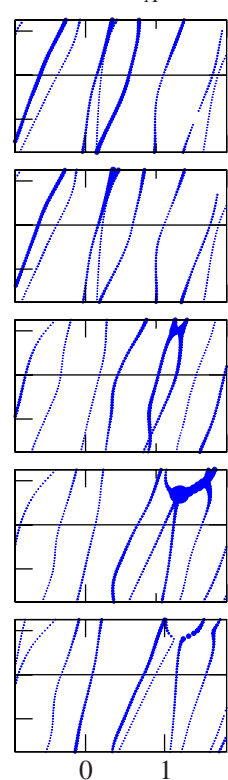

$k_{\perp}\left(\AA^{-1}\right)$ (c) $\mu_{B}$
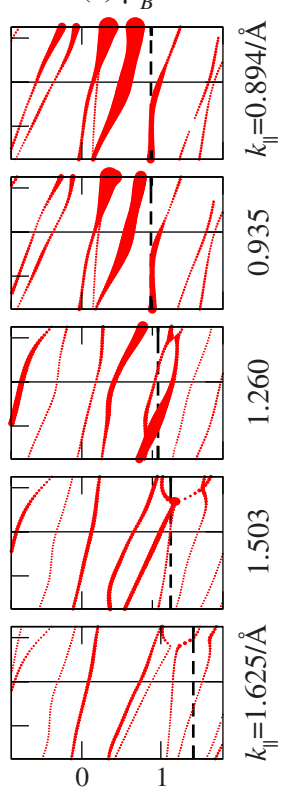

$k_{\perp}\left(\AA^{-1}\right)$
FIG. 8. (Color online) Conducting complex band structure of $\mathrm{Ni}(110)$ in the energy interval $E=93-105 \mathrm{eV}$ for five values of $k_{\|}$ between 0.894 and $1.625 \AA^{-1}$ indicated on the right side. (a) Thickness of the line is proportional to the current carried by the partial wave of the LEED function. Panels (b) and (c) show partial-wavesresolved transition amplitudes $\mu_{A}$ and $\mu_{B}$ from the initial states $\mathrm{A}$ and $\mathrm{B}$, respectively, forming the two symmetry related $\beta_{1}-\beta_{1}^{\prime}$ contours at $\epsilon=-0.5 \mathrm{eV}$, see Fig. 7(c). Thickness shows the modulus of the matrix element. Vertical dashed lines indicate $k_{\perp}$ of the incident wave B.

tion amplitude again is spread over many final-state branches but, owing to the dipole matrix element, it does not follow the distribution of the partial currents. The two upper graphs of Fig. 8(c) $\left(k_{\|}=0.894\right.$ and $\left.0.935 \AA^{-1}\right)$ demonstrate that the momentum conservation is relaxed: although the two main waves (with large $T_{\kappa}$ ) are away from the $k_{\perp}$ of the closest initial-state wave $B$ [vertical dashed lines in Fig. 8(c)] they contribute more strongly to the emission than the wave at the direct transition position because the latter has a much smaller value of $T_{\kappa}$. However, with increasing $k_{\|}$the perpendicular component $k_{\perp}$ of the closest initial-state wave moves away from the $k_{\perp}$ location of the main final-state branches and the respective partial amplitude decreases. Then the side branches with small $T_{\kappa}$ come into play and the arising direct transitions may be rather strong, see the graph $k_{\|}$ $=1.26 \AA^{-1}$ in Fig. 8(c). Also the indirect transitions to these branches are not negligible, in particular, from the initial state A located at negative $k_{\perp}$ far away from the shown finalstate waves [Fig. 8(b)].

Thus, owing to the side branches, the transition intensity changes even slower with $k_{\|}$then one would expect from a simple free-electron picture. At the same time, the $\mathbf{k}_{\|}$-projected DOS has sharp peaks [Fig. 6(d)], which give rise to the ghost features in the intensity map.

\section{CONCLUSION}

To summarize, we have reported high-resolution ARPES measurements on $\mathrm{Ni}(110)$ performed with variable polarization of synchrotron radiation. The $a b$ initio calculation of the photoemission final states confirms that with the photon energy around $100 \mathrm{eV}$, the strongest contribution to the spectra comes from the $\Gamma \mathrm{KX}$ symmetry line of the BZ, which corresponds to the direct transitions for this photon energy in the sense of minimal $k_{\perp}$ mismatch between the initial and the final state. However, apart from the spectral structures assigned to direct transitions, we have discovered ghost structures with pronounced polarization dependence which could not be attributed to any states at the ГKX line. Based on the one-step photoemission theory, we have found that these structures are a result of the intrinsic $k_{\perp}$ broadening of the final states, which invokes signal from initial states situated away from the $\Gamma \mathrm{KX}$ line but having large $\mathbf{k}_{\|}$-projected DOS. Despite relatively high photon energy, in some regions of $k$ space the final states significantly deviate from the freeelectron parabola and include a few Bloch waves, each effectively coupling to the photoelectron plane wave.

Being a transition metal, nickel is an example of a system that for the photoemission analysis presents an intermediate case between simple metals such as aluminum ${ }^{19}$ and $2 \mathrm{D}$ structures such as many high- $T_{c}$ superconductors. In simple metals, the DOS $\rho\left(\mathbf{k}_{\|}, \boldsymbol{\epsilon}\right)$ is not reflected in the spectra, and the spectral structures are determined by (approximately) direct transitions. In 2D systems, on the contrary, the spectrum reflects sharp peaks in $\rho\left(\mathbf{k}_{\|}, \epsilon\right)$, and the only role of the final state is to modify their intensity. In the present experiment on $\mathrm{Ni}$ we have seen a combination of the two pictures: the photoemission peaks originate from the peaks in $\rho\left(\mathbf{k}_{\|}, \epsilon\right)$ with the states around $k_{\perp}=0$ strongly enhanced and those around $k_{\perp}$ $= \pm 2 \sqrt{2} \pi / a$ entirely damped due to the effect of the final states. This causes the asymmetric structure of the MDCs. The DOS features coming from $k_{\perp}$ around $\sqrt{2} \pi / a$ give rise to the ghost structures, which are a manifestation of indirect transitions.

A detailed knowledge of final states is important for the extraction of self-energy information from the photoemission measurement. ${ }^{18}$ The present study shows that the measurement of the ghost structures can serve to verify and refine the one-step model of photoemission, thereby providing a more reliable estimate of the quasiparticle parameters.

\section{ACKNOWLEDGMENTS}

The measurements were done at the Surface and Interface Spectroscopy (SIS) beamline, Swiss Light Source, Paul Scherrer Institute, Villigen, Switzerland. We thank C. Hess and F. Dubi for technical support. We also thank J. LoboCheca and H. J. Dil for helpful discussion on the surface reconstruction in magnetic materials. 
*xiaoyu.cui@psi.ch

†luc.patthey@psi.ch

${ }^{1}$ T. J. Kreutz, T. Greber, P. Aebi, and J. Osterwalder, Phys. Rev. B 58, 1300 (1998).

${ }^{2}$ M. Higashiguchi, K. Shimada, K. Nishiura, X. Cui, H. Namatame, and M. Taniguchi, Phys. Rev. B 72, 214438 (2005).

${ }^{3}$ A. Hofmann, X. Y. Cui, J. Schäfer, S. Meyer, P. Höpfner, C. Blumenstein, M. Paul, L. Patthey, E. Rotenberg, J. Bünemann, F. Gebhard, T. Ohm, W. Weber, and R. Claessen, Phys. Rev. Lett. 102, 187204 (2009).

${ }^{4}$ C. S. Wang and J. Callaway, Phys. Rev. B 15, 298 (1977).

${ }^{5}$ F. Weling and J. Callaway, Phys. Rev. B 26, 710 (1982).

${ }^{6}$ I. V. Solovyev and M. Imada, Phys. Rev. B 71, 045103 (2005).

${ }^{7}$ S. Biermann, F. Aryasetiawan, and A. Georges, Phys. Rev. Lett. 90, 086402 (2003).

${ }^{8}$ J. Bünemann, F. Gebhard, T. Ohm, R. Umstätter, S. Weiser, W. Weber, R. Claessen, D. Ehm, A. Harasawa, A. Kakizaki, A. Kimura, G. Nicolay, S. Shin, and V. N. Strocov, Europhys. Lett. 61, 667 (2003).

${ }^{9}$ J. Braun, J. Minár, H. Ebert, M. I. Katsnelson, and A. I. Lichtenstein, Phys. Rev. Lett. 97, 227601 (2006).

${ }^{10}$ J. Schäfer, D. Schrupp, E. Rotenberg, K. Rossnagel, H. Koh, P. Blaha, and R. Claessen, Phys. Rev. Lett. 92, 097205 (2004).

${ }^{11}$ X. Y. Cui, K. Shimada, Y. Sakisaka, H. Kato, Y. Aiura, M. Higashiguchi, Y. Miura, H. Namatame, and M. Taniguchi, Physica B 383, 146 (2006).

${ }^{12}$ X. Y. Cui, K. Shimada, M. Hoesch, Y. Sakisaka, H. Kato, Y. Aiura, S. Negishi, M. Higashiguchi, Y. Miura, H. Namatame, and M. Taniguchi, J. Magn. Magn. Mater. 310, 1617 (2007).

${ }^{13}$ X. Y. Cui, K. Shimada, M. Hoesch, Y. Sakisaka, H. Kato, Y. Aiura, M. Higashiguchi, Y. Miura, H. Namatame, and M. Taniguchi, Surf. Sci. 601, 4010 (2007).

${ }^{14}$ H. A. Mook and D. McK. Paul, Phys. Rev. Lett. 54, 227 (1985).

${ }^{15}$ A. T. Boothroyd, T. G. Perring, A. D. Taylor, D. McK. Paul, and H. A. Mook, J. Magn. Magn. Mater. 104-107, 713 (1992).

${ }^{16}$ V. N. Strocov, E. E. Krasovskii, W. Schattke, N. Barrett, H. Berger, D. Schrupp, and R. Claessen, Phys. Rev. B 74,
195125 (2006).

${ }^{17}$ E. E. Krasovskii, V. N. Strocov, N. Barrett, H. Berger, W. Schattke, and R. Claessen, Phys. Rev. B 75, 045432 (2007).

${ }^{18}$ E. E. Krasovskii, K. Rossnagel, A. Fedorov, W. Schattke, and L. Kipp, Phys. Rev. Lett. 98, 217604 (2007).

${ }^{19}$ E. E. Krasovskii, W. Schattke, P. Jiříček, M. Vondráček, O. V. Krasovska, V. N. Antonov, A. P. Shpak, and I. Bartoš, Phys. Rev. B 78, 165406 (2008).

${ }^{20}$ R. Matzdorf, Appl. Phys. A: Mater. Sci. Process. 63, 549 (1996).

${ }^{21}$ V. N. Strocov, J. Electron Spectrosc. Relat. Phenom. 130, 65 (2003).

${ }^{22}$ S. Hüfner, Photoelectron Spectroscopy, 3rd ed. (Springer-Verlag, Berlin, 2003), and references therein.

${ }^{23}$ W. Eberhardt and E. W. Plummer, Phys. Rev. B 21, 3245 (1980).

${ }^{24}$ J. Bünemann, F. Gebhard, T. Ohm, S. Weiser, and W. Weber, Phys. Rev. Lett. 101, 236404 (2008).

${ }^{25}$ M. Mulazzi, M. Hochstrasser, M. Corso, I. Vobornik, J. Fujii, J. Osterwalder, J. Henk, and G. Rossi, Phys. Rev. B 74, 035118 (2006).

${ }^{26}$ L. Eierdal, F. Besenbacher, E. Laegsgaard, and I. Stensgaard, Surf. Sci. 312, 31 (1994).

${ }^{27}$ A. M. Baró, G. Binnig, H. Rohrer, Ch. Gerber, E. Stoll, A. Baratoff, and F. Salvan, Phys. Rev. Lett. 52, 1304 (1984).

${ }^{28}$ P. J. Feibelman and D. E. Eastman, Phys. Rev. B 10, 4932 (1974).

${ }^{29}$ E. E. Krasovskii, F. Starrost, and W. Schattke, Phys. Rev. B 59, 10504 (1999).

${ }^{30}$ E. E. Krasovskii and W. Schattke, Phys. Rev. B 56, 12874 (1997).

${ }^{31}$ J. C. Slater, Phys. Rev. 51, 840 (1937).

${ }^{32}$ V. Heine, Proc. Phys. Soc. 81, 300 (1963).

${ }^{33}$ The concept of absorbed currents was introduced in V. N. Strocov, H. I. Starnberg, and P. O. Nilsson, Phys. Rev. B 56, 1717 (1997); and a detailed explanation has been presented in N. Barrett, E. E. Krasovskii, J.-M. Themlin, and V. N. Strocov, ibid. 71, 035427 (2005). 